am 27.1.2003 stattgefunden haben, während die konkrete Behandlung durch die Beschuldigte bereits etliche Tage vorher, nämlich mit der Bestellung des Schnittpräparats bei den Pathologen, begonnen hatte; am 26.1.2003 waren der Zapper und das Schnittpräparat den Angehörigen bereits ausgehändigt worden.

Vor diesem Hintergrund kann die Frage offen bleiben, ob und in welchem Umfang die Aufklärungspflicht, die zu den wesentlichen Kernpflichten der ärztlichen Tätigkeit gehört, in einer von Eheleuten betriebenen Gemeinschaftspraxis delegierbar ist (vgl. allgemein zur Delegation der Aufklärung Wenzel, in: Wenzel [Hrsg.], Handbuch des Fachanwalts Medizinrecht, 2. Aufl. 2009, Kap. 4, Rdnr. 151 und Rdnrn. 817 ff.; Terbille, in: Terbille [Hrsg.], Münchener Anwaltshandbuch Medizinrecht, 2009, \$1, Rdnr. 389).

Ebenso wenig musste der Senat der sich aufgrund der besonderen Umstände aufdrängenden Frage nachgehen, warum die angeblich schon vor Jahren angefertigten handschriftlichen Vermerke des Herrn Dr. U. erst zwei Tage vor der Hauptverhandlung vorgelegt wurden, oder näher aufklären, warum es ausgerechnet zur Frage der Aufklärung in ganzen Sätzen ausformulierte Erklärungen gibt, während sämtliche sonstigen Einträge in den - ebenfalls vorgelegten - Krankenblättern ausnahmslos stichwortartig verfasst sind.

f) Schließlich hat das Berufsgericht zu Recht zulasten der Beschuldigten auf die enorme Schädigung des Ansehens der Ärzteschaft hingewiesen, die durch die umfangreiche Presseberichterstattung bewirkt wurde. Dass es hierbei zu teilweise unsachlichen Darstellungen gekommen ist, ist zu bedauern, muss aber als eine in vergleichbaren Situationen häufig vorkommende Begleiterscheinung betrachtet werden. Entgegen den wiederholten Behauptungen der Beschuldigten ist allerdings nicht erkennbar, dass unrichtige Mitteilungen an die Presse von der Antragstellerin ausgegangen sind.

g) Dennoch hat der Senat die teilweise sensationsgierige Darstellung der Beschuldigten in der Boulevardpresse sowie die Dauer des gerichtlichen Verfahrens in Grenzen mildernd berücksichtigt. Ebenso hat er der Beschuldigten zugute gehalten, dass sie berufsrechtlich nicht vorbelastet ist.

B. Die Berufung der Beschuldigten ist aus den Gründen zu A. ebenfalls zulässig, aber unbegründet.

BUCHBESPRECHUNGEN

DOI: 10.1007/s00350-010-2701-6

\section{Krankenhausrecht. Praxishandbuch zum Recht des Krankenhauswesens.}

Herausgegeben von Stefan Huster und Markus Kaltenborn. Verlag C.H. Beck, München 2010, XXXI u. 606 S., Ln., $€ 135,00$

Ein untrügliches Zeichen dafür, dass einzelne Gruppen von Rechtsnormen zu einem eigenständigen abgrenzbaren Rechtsgebiet zusammengewachsen sind, ist die Entstehung eines Handbuchs. Beim Krankenhausrecht ist es schon seit langem so weit: Aus einer Vielzahl von bundes- und landesrechtlichen, zivil- und öffentlich-rechtlichen Bestimmungen bestehend, hat es sich zu einer etablierten Materie der Teilrechtsordnung gemausert. Im Unterschied zu der eher zivilrechtlich geprägten Darstellung im von Laufs/Kern herausgegebenen Handbuch des Arztrechts stehen in dem hier anzuzeigenden „Bochumer Handbuch“ die öffentlich-rechtlichen Implikationen des Rechtsgebiets im Vordergrund. Eine solche zusammenhängende Darstellung fehlte bislang, war aber eigentlich überfällig. Denn das Krankenhausrecht zeichnet sich durch einige Besonderheiten aus, die die Zugänglichkeit der Materie erschweren und daher eine systematische Aufbereitung nahe legen. Im Schnittfeld zwischen Sozialversicherungsrecht und staatlichem Planungsrecht angesiedelt, ist es zwar weniger stark korporatistisch geprägt als das Vertragsarztrecht. Aber ebenso wie in anderen Versorgungsbereichen (z. B. Hilfsmittelrecht, möglicherweise demnächst auch Arzneimittelpreisrecht) gewinnt das (Kollektiv-)Vertragsrecht zunehmend an Bedeutung, vor allem durch die Reform des Vergütungsrechts.

Das Handbuch arbeitet nach einführenden Kapiteln zu den wirtschaftswissenschaftlichen $(\$ 1$, Wasem/Walendzik/Rotter $)$, verfassungsrechtlichen $(\$ 2$, Kaltenborn) und europarechtlichen Grundlagen $(\$ 3$, Cremer, mit ausführlicher Behandlung insbesondere der beihilferechtlichen Problematik des kommunalen Verlustausgleichs) zunächst das Krankenhausrecht im engeren Sinne auf. Etwa ein Drittel

Prof. Dr. iur. Thorsten Kingreen,

Regensburg, Deutschland der Darstellungen entfällt auf die Kapitel zur Krankenhausplanung (Stollmann, \4) und -finanzierung (Degener-Hencke, \5) sowie auf das Leistungsrecht, wobei hier zu Recht zwischen der GKV $(\$ 6$, Schrinner) und der PKV ( $\$ 7$, Patt/Wilde) differenziert wird. Konzeptionel besonders bemerkenswert ist der intradisziplinäre Zugriff, der das Krankenhausrecht in die Gesamtrechtsordnung einbindet. So werden, gewissermaßen als Krankenhausrecht im weiteren Sinne, die wettbewerbsrechtlichen Implikationen $(\$ 9$, Bold, mit einer sehr ausführlichen Darstellung des Fusions- und Kooperationsrechts) ebenso behandelt wie die steuerrechtlichen $(\$ 10$, Drüen $)$, arbeitsrechtlichen (\$11, Ricken), vertrags- und haftungsrechtlichen $(\$ 12$, Rehborn, und $\$ 13$, Gaidzik/Weimer) sowie die vergaberechtlichen $(\mathbb{S} 15$, Kaltenborn $)$ Weimer) Fragestellungen.

Eine ebenfalls bemerkenswerte Besonderheit des Handbuchs ist der grundlegende Beitrag von Vollmöller zur Krankenhausprivatisierung $(\$ 14)$ sowie der abschließende Abschnitt zu den Rechtsfragen einzelner Krankenhausträger. Hier werden die besonderen rechtlichen Rahmenbedingungen der Krankenhäuser in kommunaler (\$16A, Friedrich), freigemeinnütziger $(\$ 16 \mathrm{~B}$, Heinig/Schlüter $)$ und privater ( $\$ 16 \mathrm{C}$, Wernick) Trägerschaft ebenso behandelt wie die in einem komplizierten Schnittfeld zwischen Gesundheits- und Hochschulrecht angesiedelten Universitätskliniken (\$16D, Möller) sowie die Unfallkliniken der Berufsgenossenschaft ( $(16 \mathrm{E}$, Mehrtens/Gorn) und die Psychiatrischen Kliniken (\$16F, Kunze/Rehborn).

Konzeptionell bilden die in den letzten Jahren entstandenen und gerade entstehenden Handbücher (etwa auch zum Vertragsarztrecht und zum Arzneimittelrecht) die sektoral gegliederte Versorgungsstruktur ab. Die Grenzen zwischen den Sektoren werden aber zunehmend durchlässiger. Deshalb ist der sektorenübergreifende Beitrag von Bohle (Kooperation von Krankenhäusern mit anderen Trägern, $\$ 8)$ besonders verdienstvoll. Behandelt werden hier das Belegarztwesen, der Status von Vertragsärzten im Krankenhaus, die Integrierte Versorgung und Disease-Management-Programme speziell unter krankenhausrechtlichen Aspekten. Allerdings sollte das Kooperationskapitel noch durch ein eigenständiges „Konkurrenzkapitel“ ergänzt werden. Denn die gerade derzeit so umstrittenen Rechtsfragen im Zusammenhang mit der Erbringung ambulanter Leistungen durch Krankenhäuser werden in dem Handbuch nur gestreift.

Insgesamt kann man die Herausgeber beglückwünschen: Bochum ist in diesem Jahr nicht nur Kulturhauptstadt, sondern auch zur Hauptstadt des Krankenhausrechts geworden. 